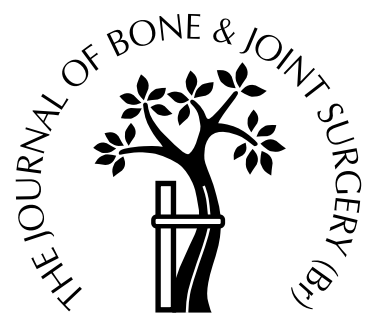

\title{
Influence of peg design and cement mantle thickness on pull-out strength of glenoid component pegs
}

\author{
R. W. Nyffeler, C. Anglin, R. Sheikh, C. Gerber \\ From the University of Zürich, Switzerland
}

$\mathbf{F}$ ixation of the glenoid component is critical to the outcome of total shoulder arthroplasty. In an in vitro study, we analysed the effect of surface design and thickness of the cement mantle on the pull-out strength of the polyethylene pegs which are considered essential for fixation of cemented glenoid components. The macrostructure and surface of the pegs and the thickness of the cement mantle were studied in human glenoid bone. The lowest pull-out forces, $20 \pm 5 \mathrm{~N}$, were for cylindrical pegs with a smooth surface fixed in the glenoid with a thin cement mantle. The highest values, $425 \pm 7 \mathrm{~N}$, were for threaded pegs fixed with a thicker cement mantle. Increasing the diameter of the hole into which the peg is inserted from 5.2 to $6.2 \mathrm{~mm}$ thereby increasing the thickness of the cement mantle, improved the mean pull-out force for the pegs tested.

J Bone Joint Surg [Br] 2003;85-B:748-52.

Received 27 June 2001;Accepted after revision 14 October 2002

One of the most common complications of total shoulder arthroplasty is loosening of the glenoid component. Radiolucent lines at the cement-bone interface are present in $30 \%$ to $96 \%$ of the patients at follow-up. ${ }^{1}$ When associated with pain and poor function, a loose component may need to be removed or replaced. Reported causes for loosening of the glenoid component are eccentric loading, conforming and constrained prosthetic systems, poor cementing technique, poor bone quality, high patient activity and infection. ${ }^{2-5}$ In an attempt to improve the fixation of a glenoid

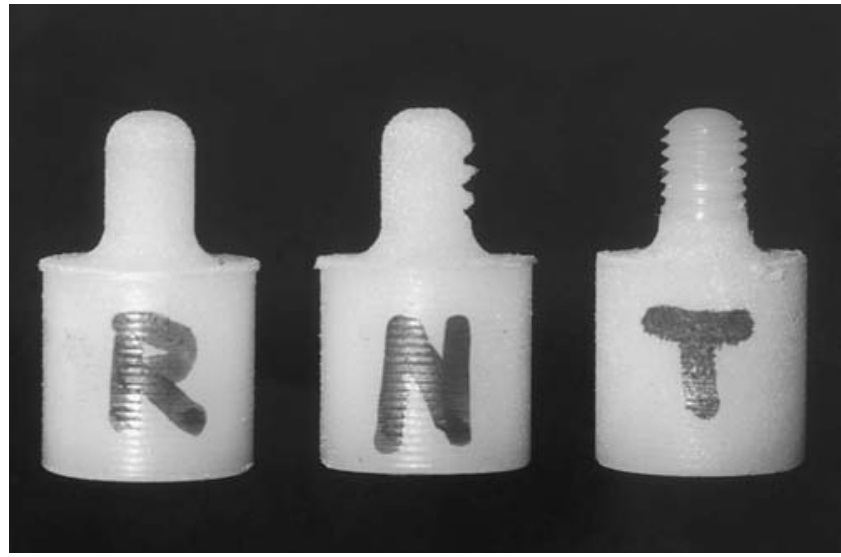

Fig. 1

Photograph showing three pegs with different macrostructures and a rough blasted surface; the diameter is $5.2 \mathrm{~mm}$ and length $8.5 \mathrm{~mm}$ (R, cylindrical; $\mathrm{N}$, notched one-sided; $\mathrm{T}$, threaded).

component in the scapular neck, keeled designs have come into competition with pegged designs. These require a large amount of cement and loosening invariably occurs between the cement and the bone. Pegged components, which need only small drill holes in the glenoid bone, normally require much less cement. The mode of failure of such components is not well known. Our aim therefore was to determine the influence of the design of the peg and the thickness of the cement mantle on the fixation strength of the pegs used for cemented fixation of glenoid components.

\section{Materials and Methods}

We subjected 48 custom-made polyethylene pegs with the shape and surface features considered necessary for the fixation of cemented glenoid components to axial pull-out tests. Each peg was $8.5 \mathrm{~mm}$ long with a diameter of $5 \mathrm{~mm}$, machined at the end of an ultra-high molecular-weight polyethylene cylinder $12 \mathrm{~mm}$ in length (Sulene; Sulzer Orthopedics Ltd, Winterthur, Switzerland). We studied the following variables in every possible combination: peg macrostructure (cylindrical, notched one-sided, threaded) as shown in Figure 1; peg surface (smooth and rough-blasted); and the diameter of the hole into which the peg is inserted 


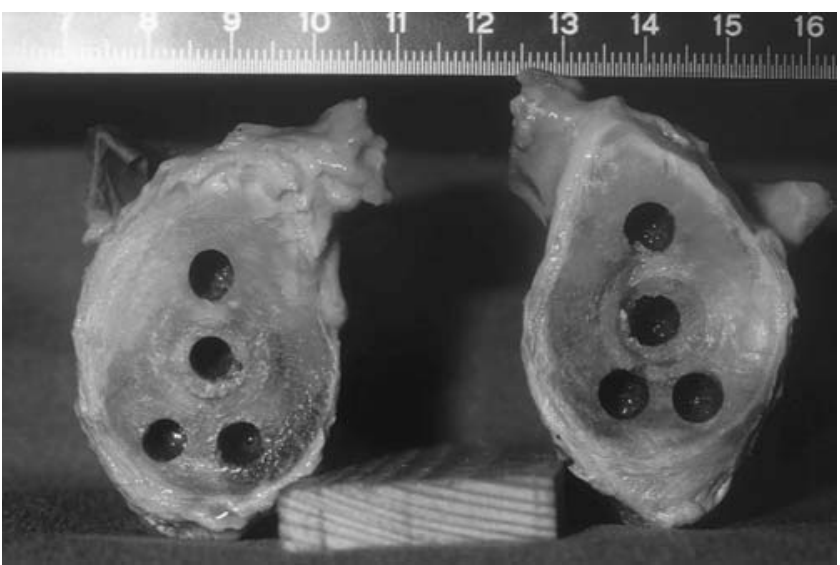

Fig. 2

Photograph showing the upper view of two cadaver glenoids used for the experiments. The diameter of the drill holes is $5.2 \mathrm{~mm}$ on the left and $6.2 \mathrm{~mm}$ on the right specimen (nominal $0.1 \mathrm{~mm}$ and $0.6 \mathrm{~mm}$ cement mantle, respectively).

(5.2 $\mathrm{mm}$, resulting in a small cement mantle and $6.2 \mathrm{~mm}$, resulting in a thicker cement mantle). The sandblasted pegs had a roughness of 4 to $6 \mu \mathrm{m}$. The threaded pegs had an inner diameter of $4.2 \mathrm{~mm}$. For each combination of parameters, four pegs were tested.

For the in vitro tests, we obtained 14 fresh-frozen human scapulae from patients of unknown age and gender. These were thawed overnight and kept moist, ready for resection of the soft tissues, acromion, coracoid process and scapular blade. Gross inspection showed no major degenerative changes of the glenoid. After fixing the scapular neck into a container $4 \mathrm{~cm}$ deep with bone cement, we removed the articular cartilage and prepared the glenoid using a spherical reamer. The subchondral bone was preserved as well as pos-

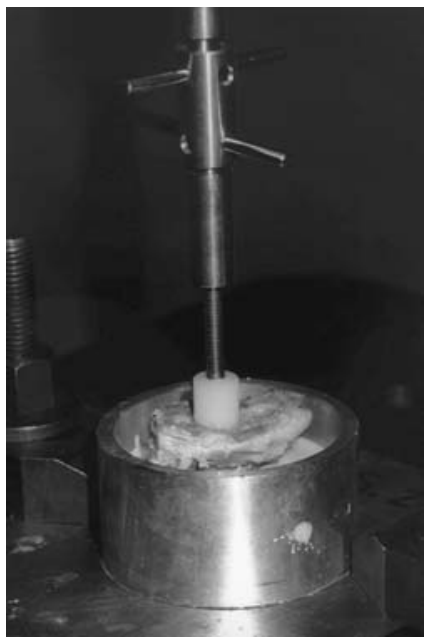

Fig. 3

Photograph showing the experimental apparatus. The polyethylene pegs were fixed perpendicular to the glenoid surface and pulled out at a constant speed of $10 \mathrm{~mm} / \mathrm{min}$. A universal joint ensured pure axial loading.

sible. Drill holes with a diameter of 5.2 or $6.2 \mathrm{~mm}$, depending on the thickness of the cement mantle being studied, and with a depth of $10 \mathrm{~mm}$, were made perpendicular to the subchondral bone, three in small specimens and four in larger specimens (Fig. 2). After irrigation with a physiological saline solution and drying with a sponge, we introduced Sulfix 60 bone cement (Sulzer Orthopedics Ltd) into each hole using a syringe. The pegs were inserted under pressure and held in position until the cement had cured. We paid special attention to placing the pegs with the same characteristics at different positions and in different bone speci-

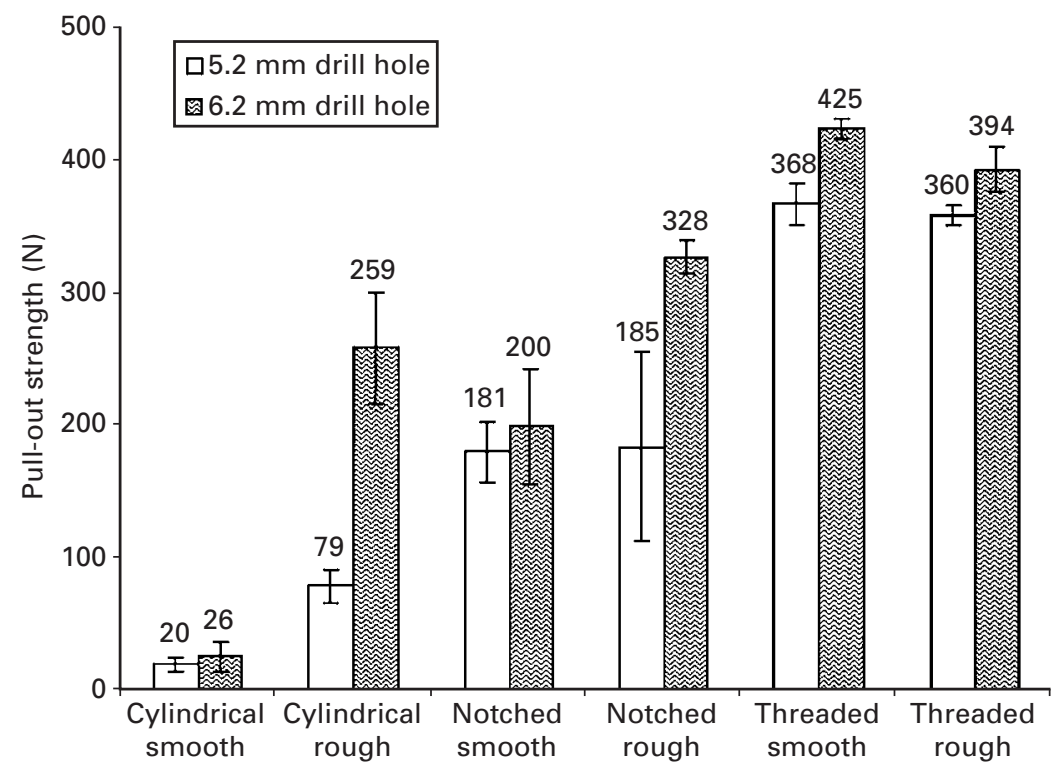

Fig. 4

Histogram showing the pull-out strength for all the pegs tested. Values are expressed as the mean and one standard deviation of the mean. 


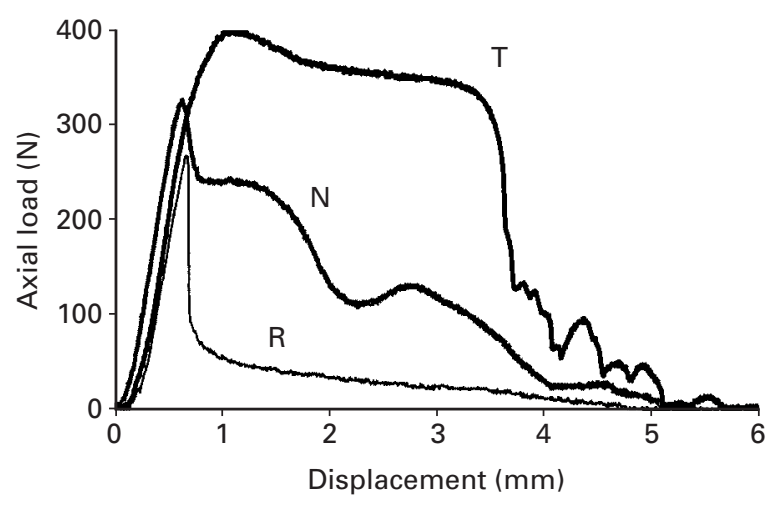

Fig. 5

Typical load-displacement curves for a cylindrical rough peg (R), a one-sided notched rough peg $(\mathrm{N})$ and a threaded rough peg $(\mathrm{T})$. The diameter of the holes into which the pegs were inserted is $6.2 \mathrm{~mm}$.

mens in order to minimise the effect of the different bone quality on the results.

An Instron 4204 universal testing machine (Instron, High Wycombe, UK) pulled the pegs out axially (Fig. 3) at a constant speed of $10 \mathrm{~mm} / \mathrm{min}$. In order to ensure pure axial loading we attached the threaded rod in the base of each cylinder to the Instron using a universal joint. A personal computer recorded the maximum pullout force and the loaddisplacement curves. We noted the mode of failure and carried out an analysis of variance using the Bonferroni-Dunn modification.

\section{Results}

For comparable surface design and thickness of the cement mantle, the threaded pegs had significantly higher pull-out strength than the notched pegs $(\mathrm{p}<0.008)$ which, in turn, had higher strength than the cylindrical pegs $(\mathrm{p}<0.006)$. The lowest pull-out forces were for cylindrical pegs with a smooth surface fixed in the glenoid bone with a thin cement mantle $(20 \pm 5 \mathrm{~N})$. As shown in Figure 4, the highest values were for threaded pegs without additional roughening of the surface fixed with a thick cement mantle $(425 \pm 7 \mathrm{~N})$.

Rough blasting of the surface resulted in a statistically significant improvement in the strength of fixation for cylindrical and notched pegs. For the threaded pegs, the surface treatment resulted in a rounding of the thread and a small reduction in pull-out strength.

Increasing the diameter of the hole into which the peg is inserted from 5.2 to $6.2 \mathrm{~mm}$ improved the mean pull-out force for all designs of peg which were tested. The difference was most obvious for rough-blasted cylindrical pegs $(259 \pm 42 \mathrm{~N} v 79 \pm 12 \mathrm{~N} ; \mathrm{p}<0.0001)$. Examination of the holes in the glenoid after the pull-out tests showed a continuous layer of cement when using a $6.2 \mathrm{~mm}$ drill (nominal $0.6 \mathrm{~mm}$ mantle) and an incomplete cement mantle when using a $5.2 \mathrm{~mm}$ drill (nominal $0.1 \mathrm{~mm}$ mantle).
Failure occurred at the polyethylene-cement interface in all cases except one. In this sample, a threaded peg failed between the cement and the bone. We observed plastic deformation of the peg in the threaded samples. The corresponding load-deformation curves showed a high pull-out resistance beyond the peak value for the threaded pegs, but not for cylindrical or notched pegs. Typical load-displacement curves for each of the three peg macrostructures tested are shown in Figure 5.

\section{Discussion}

Although loosening of the glenoid component is a common complication of total shoulder arthroplasty, there is a limited basis for selecting a particular design of glenoid. Anglin, Wyss and Pichora ${ }^{6}$ assessed the 'rocking-horse' behaviour ${ }^{2}$ of different types of glenoid components fixed with cement in a polyurethane foam bone-substitute. While the dynamic nature of this test is useful for an overall comparison, it does not have the precision, simplicity and speed of an axial pullout test. Simplicity and speed are particularly important when comparing a wide range of peg designs. Previous studies have carried out axial pull-out testing of entire glenoids (mostly keeled) or shear testing of individual pegs. ${ }^{7-9}$ If, however, we assume that the only source of tensile loading is due to compression of the humeral head on the opposite edge, which is the rocking-horse phenomenon, then only axial pull-out of individual pegs approaches the physiological situation. As translational movements of the humeral head on the glenoid component occur during active and passive glenohumeral movement, a high pull-out strength of the fixation pegs is therefore necessary for stability of the component. ${ }^{10}$

Numerous studies have been undertaken on pegs and screws designed for non-cemented fixation of tibial components, ${ }^{11-13}$ but there are none on the influence of the design of the peg and thickness of the cement mantle on the initial fixation strength. Since the number of pegged glenoid components available on the market is increasing, these factors appeared to merit study.

Previous studies have used synthetic glenoid models with consistent geometry and mechanical properties. ${ }^{6-9}$ In contrast to cadaver bone with its cancellous structure, polymeric foams have closed cells which do not allow the bone cement to flow out during insertion of the prosthesis. This may influence the pressure and therefore the friction between the glenoid model, the cement mantle and the fixation peg. We carried out our experiments on human glenoid bone. In order to reduce the influence of the quality of the bone on the results, we fixed all the pegs with the same characteristics in different specimens and in a random order.

Bone cement is chemically an ester of methacrylic acid fixed in the surrounding structures by mechanical interlocking and not by chemical bonds. ${ }^{14,15}$ Untreated, smooth polyethylene surfaces have a low coefficient of friction and pulled out of the cement very easily in our experiments. 


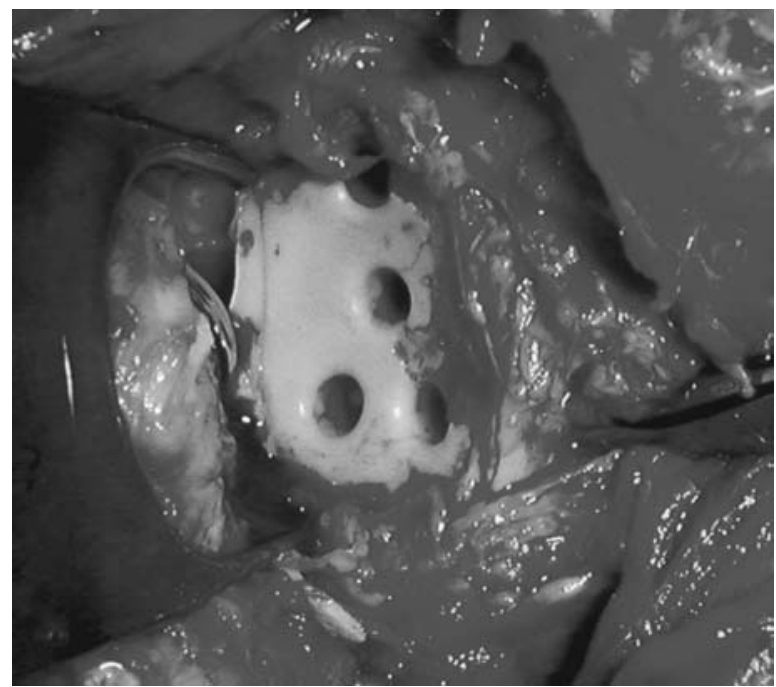

Fig. 6

Photograph showing the intraoperative view of a right shoulder after removal of a loose glenoid component with smooth fixation pegs. Failure occurred between the fixation pegs and the cement, which is still attached to the bone.

Roughening of the surface, e.g. by sandblasting, increased the friction with the adjacent bone cement and significantly increased the strength of fixation. An additional macrostructure, such as notches or threads, further increased both the mechanical interlocking with the cement and the pull-out strength in our experiments.

We tested two different sizes of hole. A hole whose diameter is only slightly larger than the diameter of the peg gives guidance in positioning and a near press-fit of the glenoid component. The disadvantage found was that the cement layer was thin and possibly incomplete circumferentially, leading to fracture of the cement and easy pull-out of the peg.

Cemented glenoid components with a keel normally loosen in vivo between the cement and bone. The mode of failure of glenoid components with pegs is not well known. The mode of failure in our experiments was primarily due to the inferior hold between the polyethylene peg and cement mantle compared with that between cement and bone. Close inspection revealed extrusion of the peg from within the cement to the extent that even a threaded surface finally disengaged itself from the surrounding cement under the action of a tensile force, which gave rise to cold flow of the polyethylene. In contrast to this loosening mechanism, the cement plug itself was not only well anchored by interlocking with the surrounding trabeculae, but also by the resistance offered by the firm plate of subchondral cortical bone. Conditions in vivo may be different since, instead of a continuous tensile force, erratic alterations of force in magnitude and direction occur. This may give rise to bone resorption and loosening between the cement and bone, and therefore, not to the loosening mechanism which we observed in vitro. Nevertheless, loosening between poly-

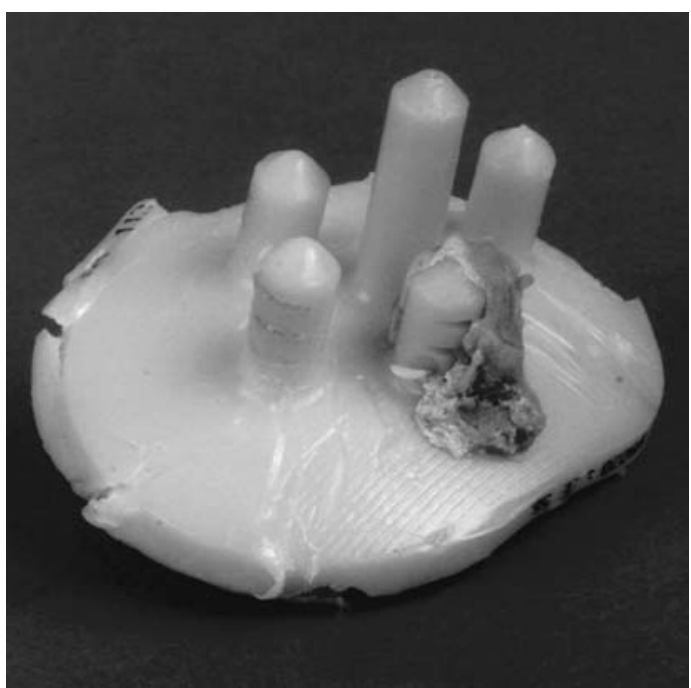

Fig. 7

Photograph showing the typical appearance of a pegged polyethylene glenoid component which was removed because of aseptic loosening. Although all peripheral pegs had small notches, failure occurred between polyethylene and cement in four of the five pegs.

ethylene and cement also occurs in patients implanted with pegged glenoid components from different manufacturers (Figs 6 and 7).

We have not changed the length or the diameter of the pegs. It seems logical that with longer or larger pegs the pull-out strength could increase. We chose the length of peg according to the availability of glenoid bone and $8.5 \mathrm{~mm}$ appeared to be reasonable in man. Thicker pegs would require a smaller number, since there is a danger of fracture between the holes. As this may weaken the anchorage, thicker pegs were not tested.

In vitro pull-out strength of cemented pegs for the fixation of polyethylene glenoid components in total shoulder arthroplasty therefore depends on the surface of the peg and the thickness of the cement mantle. A macrostructure on the peg was superior to a smooth or sandblasted surface and a circular cement layer was more important than having a small hole for precise positioning of the glenoid component. A threaded macrostructure maintained a high resistance to pull-out even after the initial peak.

The authors wish to thank $\mathrm{Mr} \mathrm{N}$. Alder for his help in the preparation of the specimens and Mr H. R. Sommer for his assistance with the biomechanical testing.

One or more of the authors has received or will receive benefits for personal or professional use from a commercial party related directly or indirectly to the subject of this article.

\section{References}

1. Wirth MA, Rockwood CA Jr. Complications of total shoulderreplacement arthroplasty. J Bone Joint Surg [Am] 1996;78-A:603-16.

2. Franklin JL, Barrett WP, Jackins SE, Matsen FA 3rd. Glenoid loosening in total shoulder arthroplasty: association with rotator cuff deficiency. J Arthroplasty 1988;3:39-46.

3. Post M. Constrained arthroplasty of the shoulder. Orthop Clin North Am 1987; $18: 455-62$ 
4. Norris BL, Lachiewicz PF. Modern cement technique and the survivorship of total shoulder arthroplasty. Clin Orthop 1996;328:76-85.

5. Frich LH, Jensen NC, Odgaard A, et al. Bone strength and material properties of the glenoid. J Shoulder Elbow Surg 1997;6:97-104.

6. Anglin C, Wyss UP, Pichora DR. Mechanical testing of shoulder prostheses and recommendations for glenoid design. J Shoulder Elbow Surg 2000;9:323-31.

7. Fukuda K, Chen CM, Cofield RH, Chao EY. Biomechanical analysis of stability and fixation strength of total shoulder prostheses. Orthopedics 1988;11:141-9.

8. Lacaze F, Kempf KJ, Bonnomet F, Boutemy P, Colin F. Primary fixation of glenoid implants: an in vitro study. In: Walch G, Boileau P, eds. Shoulder arthroplasty. Berlin: Springer, 1999:141-6.

9. Giori NJ, Beaupre GS, Carter DR. The influence of fixation peg design on the shear stability of prosthetic implants. J Orthop Res 1990;8:892-8.
10. Harryman DTD, Sidles JA, Clark JM, et al. Translation of the humeral head on the glenoid with passive glenohumeral motion. $J$ Bone Joint Surg [Am] 1990;72-A:1334-43.

11. Blaha JD, Insler HP, Freeman MAR, Revell PA, Todd RC. The fixation of a proximal tibial polyethylene prosthesis without cement. J Bone Joint Surg [Br] 1982;64-B:326-35.

12. Finlay JB, Harada I, Bourne RB, et al. Analysis of the pull-out strength of screws and pegs used to secure tibial components following total knee arthroplasty. Clin Orthop 1989;247:220-31.

13. Volz RG, Nisbet JK, Lee RW, McMurtry MG. The mechanical stability of various noncemented tibial components. Clin Orthop 1988;226:38-42.

14. Lewis G. Properties of acrylic bone cement: state of the art review. J Biomed Mater Res 1997;38:155-82.

15. Willert HG, Mueller K, Semlitsch M. The morphology of polymethylmethacrylate (PMMA) bone cement: surface structures and causes of their origin. Arch Orthop Trauma Surg 1979;94:265-92. 